\title{
Prevalência de Cryptosporidium spp e Giardia sp em eqüinos estabulados no Jockey Club de Santa Maria - RS, Brasil
}

\author{
Prevalence of Cryptosporidium spp. and Giardia sp. infection in horses stabled in the Jockey Club of \\ Santa Maria-RS, Brazil
}

\author{
Aline Diefenbach Gomes ${ }^{\mathrm{I}}$ Clarissa Barretta ${ }^{\mathrm{I}}$ Dhalile Plein Ziegler ${ }^{\mathrm{I}}$ Lourenço Sausen $^{\mathrm{I}}$

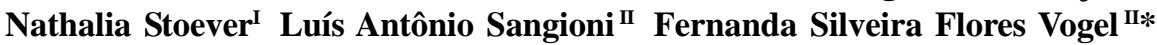 \\ Silvia González Monteiro ${ }^{\text {III }}$ Andreia Zanella ${ }^{\text {IV }}$
}

\section{- NOTA -}

RESUMO

O Cryptosporidium spp. e a Giardia sp. são atualmente reconhecidos como os principais patógenos entéricos com potencial zoonótico. O presente estudo visou estabelecer a prevalência desses protozoários em eqüinos hospedados no Jockey Club de Santa Maria, RS, Brasil, no período de 19 de maio a 30 de junho de 2007. Foram coletadas amostras de fezes, diretamente da ampola retal, de 64 animais. As amostras de fezes foram processadas por meio do método de centrifugação-flutuação de Faust modificado. Posteriormente essas amostras foram visualizadas ao microscópio óptico para a pesquisa de cistos e oocistos. Os resultados encontrados revelaram a presença de Cryptosporidium spp. em 75\% (48/64) das amostras. Cistos de Giardia sp. não foram encontrados nas amostras de fezes analisadas. A freqüência de Cryptosporidium spp. nas diferentes faixas etárias foi de 83,3\% (15/18) nos potros até dois anos de idade, $71 \%$ (22/31) nos jovens entre dois e cinco anos e $80 \%$ (12/15) nos adultos. Os resultados demonstram que o Cryptosporidium spp. está amplamente disseminado na população de eqüinos do Jockey Club de Santa Maria e pode representar uma fonte de infecção significativa para a população da região.

Palavras-chave: protozoários, epidemiologia, zoonose.

\begin{abstract}
Cryptosporidium spp. and Giardia sp. are currently recognised as the main enteric pathogens with potential zoonotic transmission risk. The present study aimed to investigate the prevalence of these parasites in horses stabled in the Santa Maria Jockey Club between May 19 and June 30, 2007. Fecal samples from 64 horses were collected directly from the animals' rectal ampoule. The 64 fecal samples were processed using modified Faust's method through the centrifugation-floatation technique, and were then later visualized under optical microscope for detection of Cryptosporidium spp. oocysts and Giardia sp. cysts. The results showed the occurrence of Cryptosporidium spp. in 75\% (48/64) of the samples. Giardia sp. cysts were not found in the fecal samples analysed. The prevalence of Cryptosporidium spp. in foals below two years of age was $83.3 \%(15 / 18) ; 71 \%(22 / 31)$ in young foals aged between three and five years of age, and $80 \%$ (12/15) in adult horses. These results show that Cryptosporidium spp. is widely disseminated in this population, and it can represent an important source of infection for the population in the region.
\end{abstract}

Key words: Cryptosporidium spp., Giardia sp., horses, occurrence.

Os protozoários Cryptosporidium spp. e Giardia sp. são reconhecidos como agentes primários

ICurso de Medicina Veterinária, Universidade Federal de Santa Maria (UFSM), Santa Maria, RS, Brasil.

IIDepartamento de Medicina Veterinária Preventiva (DMVP), Centro de Ciências Rurais (CCR), UFSM. Laboratório de Doenças Parasitárias. Avenida Roraima, nº 1000, sala 5149, prédio 44, Bairro Camobi, 97105-900, Santa Maria, RS, Brasil. E-mail: fervogel@smail.ufsm.br. *Autor para correspondência.

IIIDepartamento de Parasitologia e Microbiologia, Centro de Ciências da Saúde (CCS), Universidade Federal de Santa Maria (UFSM), Santa Maria, RS, Brasil.

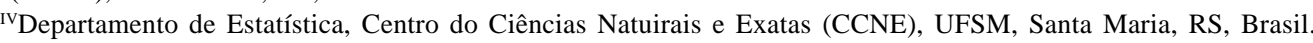


de problemas intestinais em seres humanos e animais (JOHNSON, 1997). A severidade da doença é diretamente proporcional ao estado imunológico do hospedeiro envolvido, sendo por isso de importância especial para indivíduos com a Síndrome da Imunodeficiência Adquirida (FEELY, 1990). A transmissão desses parasitas pode ocorrer pela via fecal-oral ou por meio da ingestão de água e alimentos contaminados (JOHNSON, 1997), sendo influenciada pelo nível de contaminação ambiental, pela sobrevivência da forma infectante às condições do meio (ROBERTSON et al., 1992) e pela resistência do oocisto aos métodos usados no tratamento da água como a cloração, a ozonização e a filtração (KORICH et al., 1990).

KIRKPATRICK (1989) verificou nos Estados Unidos que 65 a 97\% das águas de superfície estão contaminadas com oocistos de Cryptosporidium spp. e Giardia sp. Já BJORNEBY (1991) relatou a morte de cinco potros com imunodeficiência severa devida à infecção por Cryptosporidium spp., demonstrando a severidade da infecção nesses animais.

O curso da infecção pelo Cryptosporidium spp. geralmente é autolimitante em indivíduos imunocompetentes. No entanto, em animais imunossuprimidos, o Cryptosporidium spp. é reconhecido como um microrganismo patogênico determinando diarréia aquosa, má absorção e perda de peso (JOHNSON, 1997). A redução na taxa de crescimento, a piora da conversão alimentar ou a perda de peso, conforme observado por OLSON et al.. (1995), podem ocorrer nos eqüinos infectados, apesar de KIRKPATRICK \& SKAND (1985) relatarem que animais infectados com estes parasitas raramente manifestam sinais associados com diarréia, letargia e anorexia. A freqüência de infecções por Cryptosporidium spp. em eqüinos é pouco conhecida e acredita-se que a maioria dos animais infectados sejam portadores assintomáticos (OLSON, et al. 1997). Entre as técnicas coproparasitológicas, a concentração e a flutuação de fezes (Faust modificado) é a mais indicada para o diagnóstico de oocistos de Cryptosporidium spp. e Giardia sp. (HUBER et al., 2003; HUBER et al., 2004). Devido à escassez de dados referentes à prevalência destes protozoários nessa espécie, é preciso buscar mais informações sobre a prevalência destes parasitas. Além disso, ressalta-se a importância desse protozoário em saúde pública, uma vez que TRAUB et al. (2005) concluíram que o contato direto durante o trabalho ou a recreação com eqüinos infectados por Giardia sp. e Cryptosporidium spp. pode constituir uma fonte importante de infecção para seres humanos.
O trabalho foi realizado no Jockey Club de Santa Maria (JCSM) durante o período de 19 de maio a 30 de junho de 2007. As análises laboratoriais foram realizadas nos laboratórios de Parasitologia e Doenças Parasitárias da Universidade Federal de Santa Maria (UFSM). A pesquisa envolveu a totalidade de eqüinos hospedados nas dependências do JCSM. Foram utilizados 66 animais, entre dois e 12 anos, machos e fêmeas, sendo a maioria da raça Puro Sangue Inglês (52/64). Amostras de fezes foram coletadas diretamente da ampola retal. Depois elas foram identificadas e acondicionadas em caixas de isopor com gelo reciclável e enviadas ao laboratório para processamento. Para verificação da prevalência de Cryptosporidium spp. e Giardia sp., as amostras foram submetidas à técnica de Faust modificado, concentração pela centrífugoflutuação com sulfato de zinco (densidade específica = $1.180 \mathrm{~kg} \mathrm{~m}^{-3}$ ). Para tentar estabelecer alguma diferença na freqüência de detecção quanto à idade, os animais foram classificados em: potros (8 meses a 2 anos), jovens ( 3 a 5 anos) e adultos (seis a 12 anos); e também quanto ao sexo. Os eqüinos não apresentavam diarréia ou qualquer outra alteração clínica.

Os animais recebiam ração concentrada duas vezes ao dia em cochos de cimento fixados no interior das cocheiras e feno de alfafa fornecido no chão, uma vez ao dia. Alguns eqüinos recebiam cenoura como complemento alimentar. Aágua era fornecida ad libidum em bebedouros com bóias de nível. O sal mineral era fornecido uma vez por semana na forma granulada no comedouro. As camas eram de maravalha ou palha de arroz e foram trocadas semanalmente. As fezes eram removidas das camas, duas vezes ao dia, e depositadas em esterqueiras, situadas próximas às cocheiras, no período matutino e vespertino. Alguns animais pastavam poucas horas em piquete de campo nativo, próximo às cocheiras. Além dos eqüinos do JCSM, outros animais de carroceiros da comunidade tinham acesso a esses piquetes. Os dados dos animais foram anotados e analisados. Para a análise estatística, foi utilizado o software SAS V8. A ocorrência nas faixas etárias foi analisada por meio do teste de Qui Quadrado e entre os sexos a ocorrência foi analisada pelo teste de Fisher, com 95\% de intervalo de confiança.

Após a realização da técnica de Faust modificada, constatou-se a presença de oocistos de Cryptosporidium spp. em 75\% (48/64) das amostras das fezes dos eqüinos, tendo nos potros uma prevalência de 77,7\% (14/18), nos jovens de 71\% (22/ 31) e nos adultos de $80 \%$ (12/15). Não foi evidenciada presença de cistos de Giardia sp. nas amostras analisadas. 
A prevalência de Cryptosporidium spp. foi considerada alta (75\%, 48/64) em relação aos resultados obtidos por JOHNSON (1997), que demonstraram a ausência de oocistos. Provavelmente esses índices são atribuídos à água contaminada com oocistos infectantes, sugerindo grande contaminação hídrica no JCSM. Não houve diferença estatística na prevalência dos potros (77,7\% 14/18), dos animais jovens (71\% 22/ 31) e dos animais adultos (80\% 12/15). Esses resultados discordam com os de XIÃO (1994), que mostrou a maior prevalência em potros e animais imunodeprimidos. OLSON et al. (1997) encontraram uma maior prevalência em eqüinos maiores de 6 meses de idade. Quanto à variável sexo, não foram observadas diferenças estatísticas, sendo que a prevalência encontrada em machos foi de 75,6\% (31/41) e nas fêmeas de 76,2\% (16/ 21).

MEIRELES et al. (2007) relataram que as capivaras adultas excretam menor quantidade de oocistos e de forma intermitente. GREEN et al. (2004) demonstraram que ovinos adultos excretam pequena quantidade de oocistos no meio ambiente e de maior intensidade durante ou logo após as estações chuvosas. No Rio Grande do Sul, o outono difere das demais regiões brasileiras, pois apresenta temperaturas mais amenas e de maior precipitação hídrica, o que possivelmente pode ter favorecido condições ambientais para o desenvolvimento de formas infectantes do protozoário.

Quanto à prevalência de Giardia sp., XIÃO \& JOHNSON (1994) demonstraram que geralmente há incidência concomitante de Cryptosporidium spp. e Giardia sp. em eqüinos, no entanto, neste trabalho não foram detectados achados semelhantes. Neste estudo não foram encontrados resultados concordantes com o trabalho realizado por VARGAS \& RIGOLON (1998), que observaram prevalência de 21,1\% de Giardia em uma população eqüina. A ocorrência de $\boldsymbol{G}$ duodenalis nesse estudo foi menor em animais com idade menor ou igual a um ano. Estudos têm revelado a prevalência de Giardia sp. em 3,2\% dos cavalos usados para recreação na Califórnia (JOHNSON et al., 1997), 4,6\% em cavalos e mulas usados como transporte em Nevada (ATWILL et al., 2000) e 20\% em fazendas no Canadá (OLSON et al., 1997). Embora a infecção de Giardia em equinos seja considerada rara, animais de cinco a oito semanas são mais prevalentes (XIÃO \& HERD, 1994). A ausência do protozoário nesse estudo pode ser atribuída à eliminação intermitente dos cistos e à possibilidade de alguns animais encontrarem-se em período prepatente da infecção (VARGAS \& RIGOLON, 1998) ou ausência de contaminação de formas infectantes no meio ambiente. Levando em conta a escassez de estudos sobre Giardia sp. parasitando a espécie eqüina, considera-se que este relato reflete a necessidade de trabalhos que possam conflitar com os resultados encontrados.

A alta prevalência de Cryptosporidium spp. na população estudada requer estudos posteriores, envolvendo tanto a participação destes animais como fonte de infecção para os seres humanos como alguma implicação da infecção por este protozoário na performance de eqüinos atletas.

\section{REFERÊNCIAS}

ATWILL, E.R. et al. Cross-sectional study of faecal shedding of Giardia duodenalis and Cryptosporidium parvum among packstock in the Sierra Nevada Range. Equine Veterinary Journal, v.32, p.247-252, 2000.

BJORNEBY, J.M. et al. Cryptosporidium parvum merozoites share neutralization-sensitive epitopes with sporozoites. Journal Immunology, v.145, p.298-304, 1991.

FEELY, D.E. The biology of Giardia. In: MEYER, E.A. Human parasitic diseases. Amsterdan: Elsevier, 1990. V.3, p.11-50.

GREEN, R.E. et al. The seazonal distribuition of Cryptosporidium oocysts in sheep raised in the state of São Paulo. Revista Brasileira de Parasitologia Veterinária, v.13, p.125-127, 2004.

HUBER, F. et al. Comparação da eficiência da técnica de sedimentação pelo formaldeído-éter e da técnica de centrífugoflutuação modificada na detecção de cistos de Giardia sp. e oocistos de Cryptosporidium sp. em amostras fecais de bezerros. Revista Brasileira de Parasitologia Veterinária, v.12, p.135-137, 2003.

HUBER, F. et al. Comparação da eficiência da coloração pelo método da safranina a quente e da técnica de centrífugo-flutuação na detecção de oocistos de Cryptosporidium em amostras fecais de animais domésticos. Revista Brasileira de Parasitologia Veterinária, v.13, p.81-84, 2004.

JOHNSON et al. The prevalence of shedding of Cryptosporidium and Giardia spp. based on a single fecal sample collection from each of 91 horses used for backcountry recreation. Journal of Veterinary Diagnostic Investigation, v.9, p.56-60, 1997.

KIRKPATRICK C.E. et al. Susceptibility of domestic cats to infections with Giardia intestinalis cysts and trophozoites from human sources. Journal of Clinical Microbiology, v.21, p.671-680, 1989.

KIRKPATRICK C.E.; SKAND, D.L. Giardia in a horse. Journal of America Veterinary Medical Association, v.187, p.163164, 1985.

KORICH D.G. et al. Effects of ozone, chlorine dioxide, chlorine, and monochloramine on Cryptosporidium parvum oocyst viability. Applied and Environmental Microbiology, v.56, p.1423-1428, 1990.

Ciência Rural, v.38, n.9, dez, 2008. 
MEIRELLES, V.M. et al. Natural infection with zoonotic subtype of Cryptosporidium parvum in Capybara (Hydrochoerus hydrochaeris) from Brazil. Veterinary Parasitology, v.147, p.166-170, 2007.

OLSON, B.E. et al. Effects of giardiasis on production in a domestic ruminant (lamb) model. American Journal Veterinary Research, v.56, p.1470-1474, 1995.

OLSON, B.E. et al. Giardia and Cryptosporidium in Canadian farm animals. Veterinary Parasitology, v.76, p.375-381, 1997.

ROBERTSON, L.J. et al. Survival of Cryptosporidium parvum oocysts under various environmental pressures. Applied and Environmental Microbiology, v.58, p.494-497, 1992.
TRAUB, R.J. Epidemiological and molecular evidence supports the zoonotic transmission of Giardia among humans and dogs living in the same community. Veterinary Parasitology, v.128, p.253-262, 2004.

TRAUB, R.J. et al. Molecular characterization of potentially zoonotic isolates of Giardia duodenalis in horses. Veterinary Parasitology, v.130, p.317-321, 2005.

VARGAS L.; RIGOLON L.T. Ocorrência de Giardia duodenalis em eqüinos do Nordeste e Norte do Paraná/Brasil. Semina Ciências Agrárias, v.19, p.73-75, 1998.

XIAO L. Giardia infection in farm animals. Parasitology Today, v.10, p.436-438, 1994.

XIAO, L.; HERD, R.P. Epidemiology of equine Cryptosporidium and Giardia infections. Equine Veterinary Journal, v.26, p.14-17, 1994. 